Asthma

\title{
Sicherheit von Betamimetika erneut bestätigt
}

De Vries F et al. Long-acting beta2-agonists in adult asthma and the pattern of risk of death and severe asthma outcomes: a study with the General Practice Research Database. Eur Resp J. 2010 (published online, 29. März 2010)

\section{Fragestellung}

In dieser Studie wurde bei Patienten mit Asthma untersucht, ob die hausärztliche Verschreibung von lang wirksamen Beta2-Mimetika (LABA), kurz wirksamen Beta-2-Mimetika (SABA) oder inhalativen Kortikosteroiden (ICS) das Risiko, vorzeitig zu versterben, beeinflusst.

\section{Methodik}

Die Daten von ca. einer halben Million Asthmapatienten aus den beiden britischen Datenbanken „UK General Practice Research Database“ und „National Registry of Hospitalisations“" wurden ausgewertet. Es wurde ein Zeitraum von fünf Jahren analysiert. Zusätzlich wurden selektioniert Peak-Flow-Messungen durchgeführt.

\section{Ergebnisse}

Überraschenderweise verlief die Mortalitätsstatistik u-förmig, d.h. die Gesamtmortalität, die Asthmamortalität und die Hospitalisierungen wegen eines Asthmaanfalls waren in den (englischen) Asthmastufen 1 und 5 erhöht, nicht aber in den Stufen 2-4. Patienten, die besonders viele SABA, LABA, ICS und Kombinationspräparate bekamen, wiesen die höchste Mortalität auf.

Häufige Arztbesuche und eine erhöhte Asthmaexazerbationsrate fanden sich bei langjährigen Asthmapatienten mit vielen Verschreibungen häufiger als bei neu diagnostizierten Patienten und Patienten mit einer niedrigen Verschreibungsrate. Die Kombinationen von LABA oder SABA mit anderen Medikamenten, ins-

Gesamt- und Asthmamortalität in Abhängigkeit von Tabelle 1 der Krankheitsdauer und der Anzahl der Verschreibungen

\begin{tabular}{|c|c|c|c|c|c|}
\hline & \multirow{2}{*}{$\begin{array}{l}\text { Zeit seit der } \\
\text { ersten Ver- } \\
\text { schreibung }\end{array}$} & \multirow{2}{*}{$\begin{array}{l}\text { Verschreibungen } \\
\text { im vorherigen Jahr }\end{array}$} & \multicolumn{3}{|c|}{ Relatives Risiko } \\
\hline & & & SABA & LABA & ICS \\
\hline \multirow{6}{*}{$\begin{array}{l}\text { Gesamt- } \\
\text { mortalität }\end{array}$} & <3 Monate & & 3,5 & 2,1 & 2,6 \\
\hline & 3-12 Monate & & 2,1 & 1,4 & 1,7 \\
\hline & $>1 \mathrm{Jahr}$ & $<3$ Verschreibungen & Referenz & Referenz & Referenz \\
\hline & & $3-6$ Verschreibungen & 1,0 & 0,9 & 1,0 \\
\hline & & 7-12 Verschreibungen & 1,3 & 1,0 & 1,3 \\
\hline & & $>13$ Verschreibungen & 1,9 & 1,4 & 1,7 \\
\hline \multirow{6}{*}{$\begin{array}{l}\text { Asthma- } \\
\text { mortalität }\end{array}$} & $<3$ Monate & & 1,6 & 1,8 & 2,1 \\
\hline & 3-12 Monate & & 1,4 & 1,5 & 0,9 \\
\hline & $>1 \mathrm{Jahr}$ & $<3$ Verschreibungen & Referenz & Referenz & Referenz \\
\hline & & 3-6 Verschreibungen & 0,9 & 1,1 & 0,8 \\
\hline & & 7-12 Verschreibungen & 1,6 & 1,2 & 1,4 \\
\hline & & $>13$ Verschreibungen & 3,0 & 1,6 & 2,2 \\
\hline
\end{tabular}

Mit steigender Verschreibungsmenge steigen die Gesamt- und die Asthmamortalität. besondere ICS, senkten zwar das Risiko der genannten Parameter im Trend, aber nicht signifikant.

\section{Kommentar}

Prof. Dr. med.

Adrian Gillissen, Kassel

Die Studie zeigt zweierlei:

_ Je länger ein Patient an Asthma leidet und je höher sein Medikamentenverbrauch ist, desto höher ist sein Risiko, an der Erkrankung zu versterben oder einen behandlungspflichtigen Asthmaanfall zu erleiden.

— SABA und LABA führen im Vergleich zu anderen Asthmamedikamenten nicht zu einer signifikanten Steigerung der Mortalität oder von behandlungsbedürftigen Asthmaanfällen.

Datenbankanalysen haben diverse methodische Mängel. Zum Beispiel werden Asthma und COPD von Hausärzten nur mit einer Sensitivität von $70,3 \%$ und einer Spezifität von $87,7 \%$ diskriminiert. Zudem sind Mortalitätsstatistiken bezüglich der Todesursache häufig unzuverlässig. Viele Störfaktoren gehen in retrospektiven Datenbankanalysen unter, da sie nicht erkannt oder nicht spezifiziert werden können, wie die Einnahmeverlässlichkeit der Medikation, die gerade bei instabilen Asthmapatienten in praxi immer als schlecht angenommen werden muss und in dieser Untersuchung wahrscheinlich der Grund für den u-förmigen Kurvenverlauf war.

\section{Fazit}

Trotz der methodischen Schwächen bestätigt diese Analyse andere Studien. Sie zeigt, dass SABA und LABA nicht gefährlicher sind als andere Asthmamedikamente [1-4], und widerlegt die Metaanalyse von Salpeter et al. [5]. Schweres oder schwer therapierbares Asthma, d.h. ein fortgeschrittener Erkrankungsstatus und ein mangelnder Therapieerfolg, sind die wichtigsten Risikofaktoren für die Patienten und nicht die Medikamente an sich.

\footnotetext{
Literatur

1. Cochrane Database Syst Rev. 2005

2. Cochrane Database Syst Rev. 2007

3. Eur Respir J. 2009;33:21-32

4. Am J Respir Crit Care Med. 2008;178:1009-1016

5. Ann Intern Med. 2006;144:904-912
} 